\title{
Se veía venir... estallido social 18 de octubre 2019 en Chile y conflicto percibido por territorio
}

\author{
We had it coming... October 182019 social \\ outburst in Chile and perceived conflict per \\ territory
}

\author{
Mónica Alejandra Vargas Aguirre* \\ Diego Ignacio Rivera Lisboa*
}

\begin{abstract}
La constitución del orden social necesariamente involucra una dimensión espacial que es producida y produce relaciones de poder. Es así como los cambios sociales de la última mitad de siglo, en particular el colapso de un único modelo de desarrollo, también tendrían un efecto en la construcción del territorio y cómo se inscriben las relaciones de poder en el mismo. Tomando el caso de Santiago de Chile y el estallido social del 18 de octubre de 2019, este texto, toma la dimensión de conflicto de una investigación doctoral más amplia para determinar cómo la percepción de conflicto podría variar en las zonas del Gran Santiago y cómo ello es parte de las causas del estallido. El análisis de los ítems investigados previos al estallido social, muestra diferencias significativas entre el sector Oriente de Santiago y el resto de la urbe, respecto a la percepción de conflicto siendo más alta en esta última. Estas diferencias pueden ser leídas desde el aparato teórico de Pierre Bourdieu como diferencias en la doxa y la illusio de cada territorio, y estar relacionadas tanto con las nociones de alteridad respecto a los habitantes de otras zonas y los estigmas percibidos del lugar en que se habita, así como una construcción distinta respecto a las disputas por el capital y la resolución del conflicto.

Palabras clave: Conflicto, Habitus territorial, Bourdieu, estallido social
\end{abstract}

Abstract: The constitution of a social order necessarily involves a spatial
dimension which both produces and is produced by power relationships.
Thus, the social changes that have happened in the last half century, such as
the collapse of a unitary model of development, would also have an impact in
the construction of a territory and the way power relationships are inscribed

* Doctora en Ciencias Sociales, Universidad de Chile; Mg en Asentamientos Humanos y Medio Ambiente, Universidad Católica; Mg (c) Ciencia Política UAHC; Trabajadora Social Universidad de Valparaíso; Licenciada en Sociología, Universidad de Chile.e-mail: monica.vargas@ug.uchile.cl

** MsC en Digital Society, Universidad de Edimburgo; Licenciatura en Psicología; Universidad de Chile.

E-mail:diego.rivera.lisboa@gmail.com

(c) (1)

This content is licensed under a Creative Commons attribution-type BY 
on it. Using the example of Santiago de Chile and the social outburst of October 18, 2019, this text uses the conflict dimension of a broader doctoral project, to focus on how perception of conflict might vary among the zones of Santiago. An analysis of the items involved, which were previous to the social explosion, shows significant differences between the Eastern section of the city and the rest of it, with the latter showing a greater perception of conflict. These differences can be read from Pierre Bourdieu's theoretical apparatus as differences between the doxa and illusio of each territory and be associated to the construction of Otherness towards the inhabitants of the other sections of the city and the perceived stigmas that come with some places of residence, as well as with a different construction of both conflict resolution and the struggle for capital.

Keywords: Conflict, Territorial Habitus, Bourdieu, Social outburst

Recebido em 27/03/2020. Aceito em: 18/06/2020.

\section{Introducción}

La constitución del orden social en el siglo XXI y sus características han sido el foco de diversas propuestas teóricas tales como la "sociedad del riesgo" de Beck (1986), la "sociedad informacional" de Castell (1990) o la "modernidad líquida" de Bauman (2003). Estas propuestas poseen como elementos en común la noción de un aumento de complejidad en las sociedades modernas, lo que agrega dificultad para explicar "lo social" con los paradigmas sociológicos clásicos. Comparten además la idea de la imposibilidad de pensar/analizar una sociedad de manera aislada considerando la creciente conectividad y aumento de las relaciones internacionales o el advenimiento de un sistema mundo a decir de Wallerstein (2005) (2013), y, por último, estos autores ponen en el centro de sus planteamientos la idea de la inestabilidad o precarización de la vida, recalcando la figura del riesgo como un factor siempre presente en las sociedades modernas.

No obstante lo anterior, los autores difieren en diversos aspectos, lo que no permite el desarrollo de propuestas teóricas ampliamente explicativas. Para Garretón (2000) esta dificultad para describir la sociedad de la primera mitad del siglo XXI se debe a que ya no se puede hablar de una sociedad moderna, sino que dada una serie de cambios tanto políticos como económicos, se debe entender como una sociedad heterogénea de difícil clasificación. Dichos cambios, dado el colapso de los modelos desarrollistas, se mueven a un modelo con múltiples ejes de desarrollo y elementos tales como la integración social; el medio ambiente y el crecimiento económico aparecen disociados, siendo los dos primeros postergados en favor de la economía en la mayoría de los casos.

Un análisis del orden social requiere también un análisis de la dimensión territorial en tanto el espacio no existe de manera independiente a estas dinámicas, sino que, como señala Massey (1992), el espacio aparece como el momento de intersección de las relaciones sociales, algo que es al mismo tiempo constituido por lo social, y constituyente de este. Esto, para Massey (1992), necesariamente implica relaciones simbólicas y de poder. En esta línea, varios autores 
(Springer 2016, Harvey 2014, Wacquant, 2010; De Certeau, 2000; Bourdieu, 2000, Foucault, 1984;) han trabajado la relación entre el espacio, su representación y las relaciones de poder que los constituyen.

En particular, este texto se enfocará en la dimensión territorial de la percepción de conflicto como un elemento explícito de la constitución simbólica del orden social, siendo ésta enmarcada dentro de una investigación más amplia sobre la segregación socio-espacial, concentración territorial del poder y legitimación del orden social en el Gran Santiago.

El modo en que se entiende y representa el conflicto en una sociedad determinada cumple una función importante para entender el orden social de la sociedad de que se trate. De este modo, el conflicto es un componente clásico de los estudios sociológicos, siendo abordado tanto por autores clásicos como Marx o Weber, como por generaciones posteriores como Dahrendorf o Giddens, en los cuales esta noción se ha enmarcado como un elemento fundamental para articular el cambio y/o como una característica estructural de varios sistemas (Gómez, 2008). De manera concreta, estas distinciones no solo tienen consecuencias teóricas, sino que se asocian a la manera en que una sociedad se piensa a sí misma y a sus instituciones.

Teniendo lo anterior en mente, Santiago de Chile aparece como un estudio de caso interesante basado en el hecho de que la ciudad, en pocos años, ha experimentado una serie de transformaciones que la ponen en una posición particular frente a otras urbes, en tanto estas presentan en ella varios elementos que se relacionan con la distribución del poder y el territorio.

Así, por ejemplo, si bien el país presenta un crecimiento económico sostenido (Banco Mundial, 2019), este ha sido acompañado de un aumento en las cifras de desigualdad y segregación socioespacial (Banco Central Chile, 2012; SBIF, 2016; Castañeda, 2012; Facultad de Economía y Negocios, Universidad San Sebastián \& Equifax, 2016).

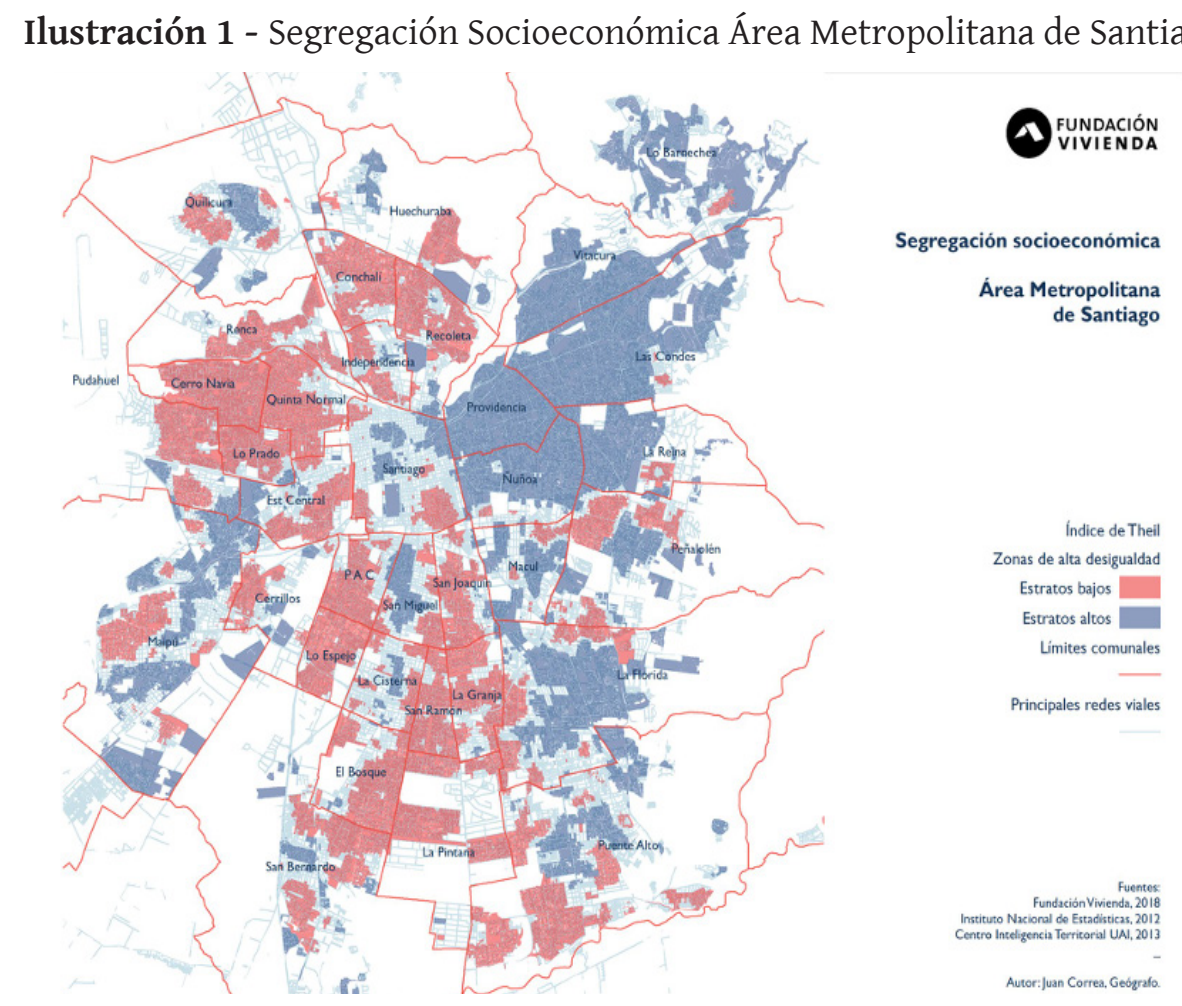

Fuente: "El Valor del suelo como reflejo y reproductor de las desigualdades" Juan Correa, geógrafo, Centro de Producción del Espacio. 
Así mismo, este periodo de crecimiento sostenido sucede tras una serie de reformas económicas instauradas en la dictadura de los 80' con el fin de reducir el rol del Estado y neoliberalizar la economía (Sepúlveda, 2016). Este proyecto, a diferencia de lo ocurrido en países como Argentina, se constituyó como el modelo hegemónico en el país sin enfrentarse a grandes desafíos que lo contrarresten, hasta las movilizaciones sociales del 2011 (Undurraga, 2015) y las posteriores movilizaciones ocurridas a partir del 18 de octubre de 2019, las cuales están evidentemente asociados a una pérdida de legitimidad de la institucionalidad neoliberal misma (Fleet, 2011; Araya, 2016) así como una permanente reducción de la participación de la ciudadanía en la estructura formal, con el consiguiente desprestigio de los partidos políticos (Araya, 2016) y el aumento de la participación de movimientos sociales específicos (Somma, 2015).

Las distinciones sociales del país se pueden observar de manera concreta a través de varios indicadores, tales como la evolución del Coeficiente de Gini desde el año 1990 hasta hoy, el que ha oscilado en torno al 0,55 (Korzeniewicz \& Moran, 2009), ubicando a Chile como el país más desigual de entre los miembros de la OCDE.

Teniendo un correlato territorial, expresado en urbes socio espacialmente segregadas, así por ejemplo, un análisis del Índice de Desarrollo Humano en el año 2003 de las comunas del Gran Santiago muestra las comunas de la zona Oriente presentan un IDH de 0,800, que de acuerdo con el PNUD (2004) sería "el valor con que se define el nivel de desarrollo humano alto a nivel comparativo internacional” (PNUD, 2004, pp. 36) es decir comparable a países de alto estándar en Europa, llegando incluso a presentar un IDH de 0,949 en el caso de la comuna de Vitacura o 0,933 en el caso de la comuna de Las Condes.

Por otro lado, comunas de la periferia norte y sur de la ciudad presentan algunos de los IDH más bajos del país, tales como Lo Espejo con un IDH de 0,657 y La Pintana con un IDH de 0,679, comparable con el INDH de países de África. Esta situación de diferencias, no varía con el pasar de los años, así, el Informe sobre Desarrollo Humano en Chile publicado por el PNUD el año 2019 señala que el país se encuentra entre las primeras 50 naciones con mejor IDH entre 186 naciones analizadas. No obstante, la desigualdad seguía siendo profunda con un Gini en torno a 4,7 situación que, según líderes de las organizaciones sociales y expertos en el tema tales como Mónica Vargas (2018), Alberto Mayol (2019), Alfredo Joignant y Mauro Basaure (2019), fue uno de los factores que provocaron el llamado "estallido social" de octubre de 2019.

Por otra parte, el 18 de octubre de 2019, estalla en Chile una gran movilización que ponen en jaque la institucionalidad democrática en el país, tanto por la violencia del mismo, como por la masividad de adscripción de la población a las demandas reivindicadas por el movimiento. Santiago se convierte entonces en el centro de masivas movilizaciones y violentos altercados entre manifestantes y policías, ante la mirada atónita de una élite que transversalmente no se explica lo que sucede y declara reiteradamente que "no lo esperaban", o "no lo veían venir", develando una profunda crisis institucional, una grieta entre la ciudadanía y la política y un absoluto distanciamiento de las elites del resto de la población.

En Santiago se plasma crudamente la desigualdad de la sociedad chilena completa. Cabe preguntarse entonces, dado el desarrollo económico desigual de los sectores de la urbe, sus niveles de segregación y las transformaciones que ha sufrido, acerca de cuál es el rol que el territorio cumple en la percepción del conflicto entre unos y otros habitantes de la ciudad, cómo podría variar entre las zonas del Gran Santiago y como ello incidió en el estallido social del 18 de octubre de 2019. 
Ilustración 2 - Índice de Desarrollo Humano Santiago de Chile

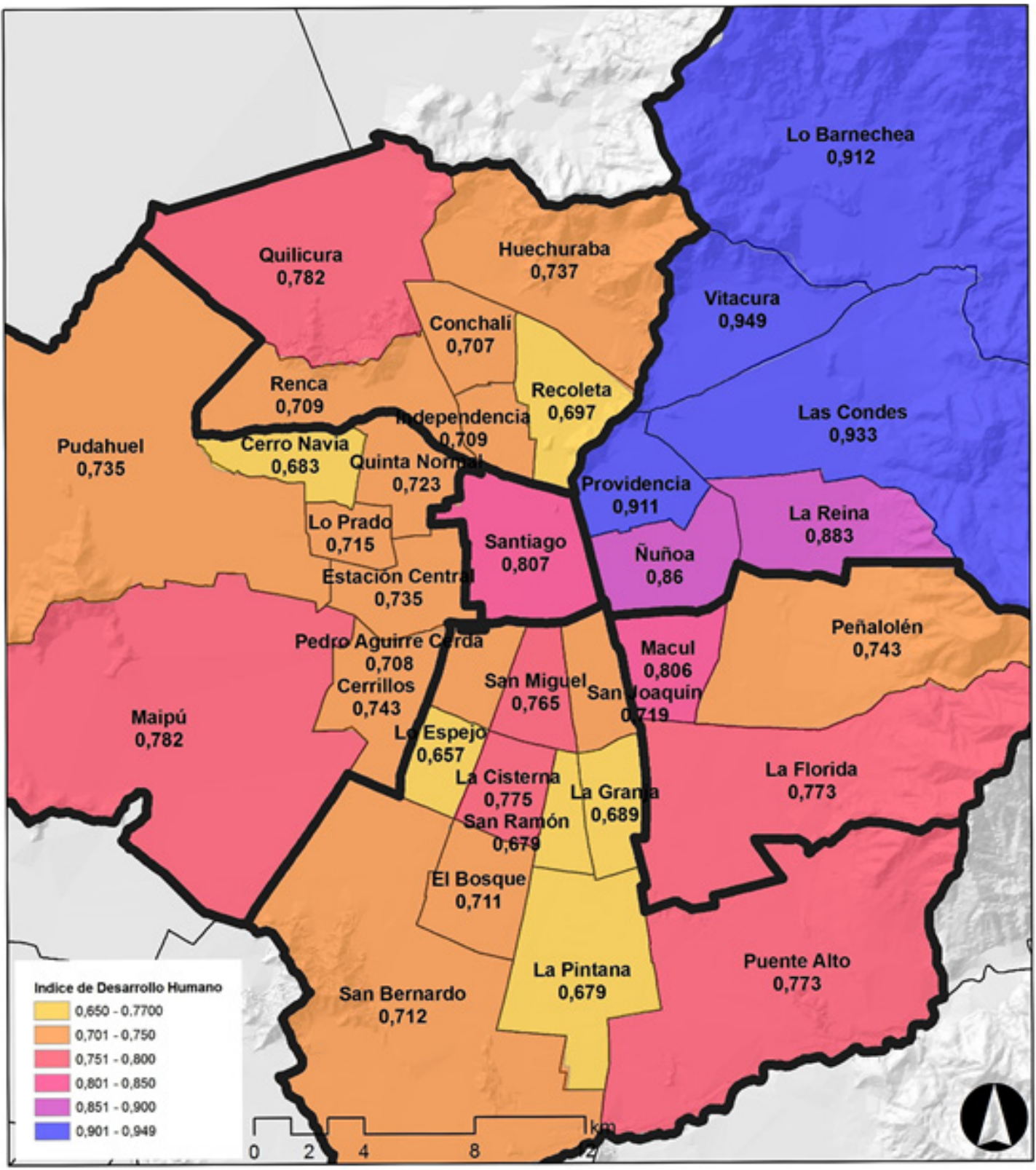

Fuente: Elaboración propia, en base a Las Trayectorias del Desarrollo Humano en las Comunas de Chile (1994-2003) (PNUD, 2004)

\section{Método}

Los datos que aquí se presentan son parte de los resultados de la investigación en el marco de la tesis doctoral denominada "Concentración territorial del poder y legitimidad del orden en Chile" cubriendo una de las dimensiones cuantitativas de los resultados de esta. La investigación recogió y analizó información en un corte temporal específico, entre 2015-2017, utilizando una escala territorial a nivel de zonas y comunas, siendo imposible trabajar a nivel de barrios o sectores específicos debido a la envergadura del estudio. Por razones similares, el área de análisis 
no abordó la totalidad del Sistema Urbano de Santiago, sino que fue circunscrito a las treinta y cuatro comunas contempladas dentro del Gran Santiago.

Para el proceso de producción de datos se produjo un cuestionario de 110 ítems, cubriendo una serie de tópicos sobre la percepción respecto a percepciones de segregación, concentración del poder y legitimidad de las instituciones. Para propósitos de este texto, se trabaja con 5 ítems relacionados con la percepción del conflicto. Como estrategia para acceder a los datos, se utilizó un muestreo aleatorio estratificado polietápico con una muestra de 480 individuos, utilizando como criterios muestrales la representación de las cinco zonas de Santiago, de acuerdo a cómo fueron caracterizadas por MIDEPLAN en el año 1991 (MIDEPLAN \& DICTUC-PUC, 1991), y Ministerio de Transporte, para los años 2001 y 2012 (Ministerio de Transporte y Telecomunicaciones, SECTRAPUC, 2001; Ministerio de Transporte y Telecomunicaciones, SECTRA-Observatorio Social, UAH, 2012) a través de la selección de una comuna por cada zona, así como la categoría de sexo y dos cohortes etarios compuestos de personas entre 20 a 24 años y personas de más de 25 años, generando una división entre aquellos que nacieron en o antes de dictadura y aquellos que no.

Los datos obtenidos por la encuesta fueron analizados en SPSS, y, a su vez, georreferenciados utilizando ArcGIS.

\section{Conflicto entre gente pobre y gente rica}

Como una manera de recoger la percepción de conflicto diferenciada por territorio es que se le preguntó a la población lo siguiente "En todos los países hay diferencias o incluso conflictos entre diferentes grupos sociales. En su opinión, ¿Cuánto conflicto hay en Chile entre: la gente pobre y la gente rica?"

Gráfico 1 - En todos los países hay diferencias o incluso conflictos entre diferentes grupos sociales. En su opinión, ¿Cuánto conflicto hay en Chile entre: la gente pobre y la gente rica?

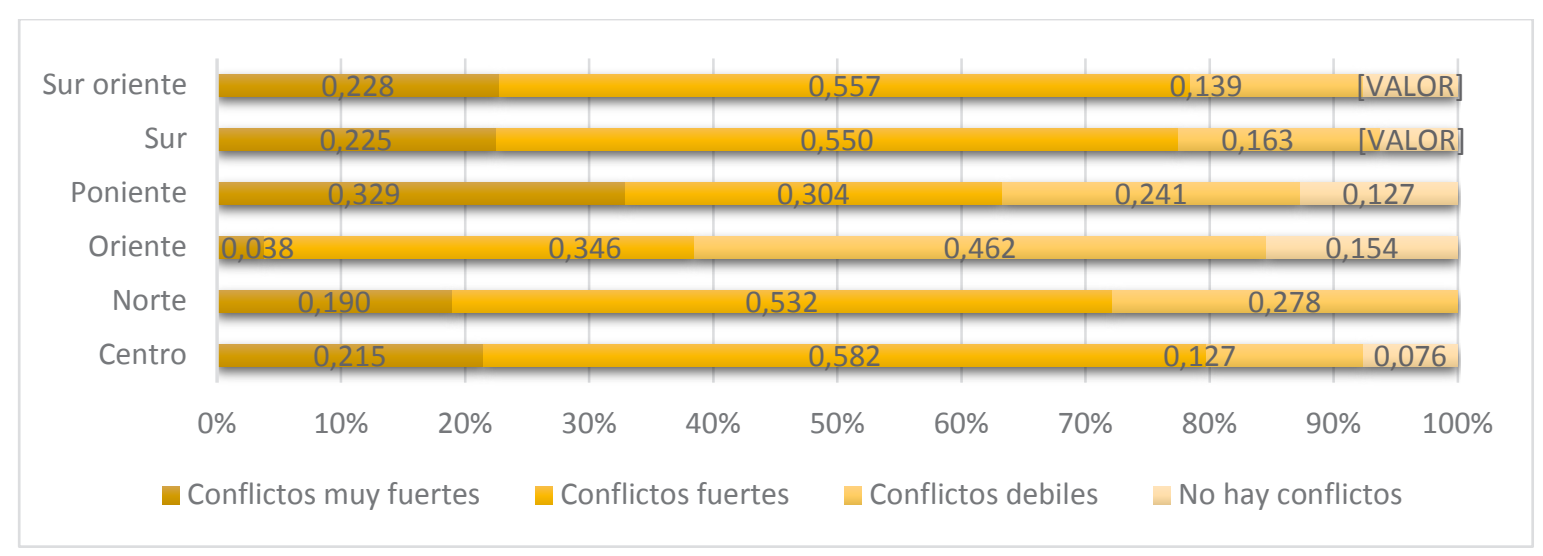

Fuente: El autor.

Esta pregunta fue tomada de la encuesta Latinobarómetro, el que mide percepciones a nivel país sobre democracia en América Latina, que en este caso se aplicó para conocer la especificidad por territorio en el gran Santiago de dicha percepción. Los resultados obtenidos son los siguientes: dentro del total de la muestra, un $67,7 \%$ cree que en Chile hay conflictos fuertes o muy fuertes entre la gente pobre y la gente rica, y solo un $8,1 \%$ cree que no hay conflicto. A nivel de zona, es importante notar que el $39 \%$ de la zona oriente considera que los conflictos entre gente pobre 
y rica son fuertes o muy fuertes, y $46 \%$ opina que son débiles, esto aparece en contraste con las otras zonas, en las cuales el $80 \%$ de la zona centro, el 79\% de los encuestados de la zona suroriente, el $78 \%$ de los de la zona sur, el $72 \%$ de la zona norte, y el 63\% de la zona poniente, opinan que en Chile hay conflictos muy fuertes o fuertes entre gente pobre y gente rica.

Esta diferencia coincide con las diferencias en grupos socioeconómicos de la urbe, en tanto las comunas de la zona oriente son en las que se concentran los grupos socioeconómicos altos y medios altos (INE, 2013), lo cual podría explicar las diferencias en este ítem en tanto sus implicancias son distintas en esta zona del territorio.

Ilustración 3 - Conflicto entre gente pobre y gente rica

\section{¿Cuánto conflicto hay en Chile entre la gente pobre y la gente rica?}

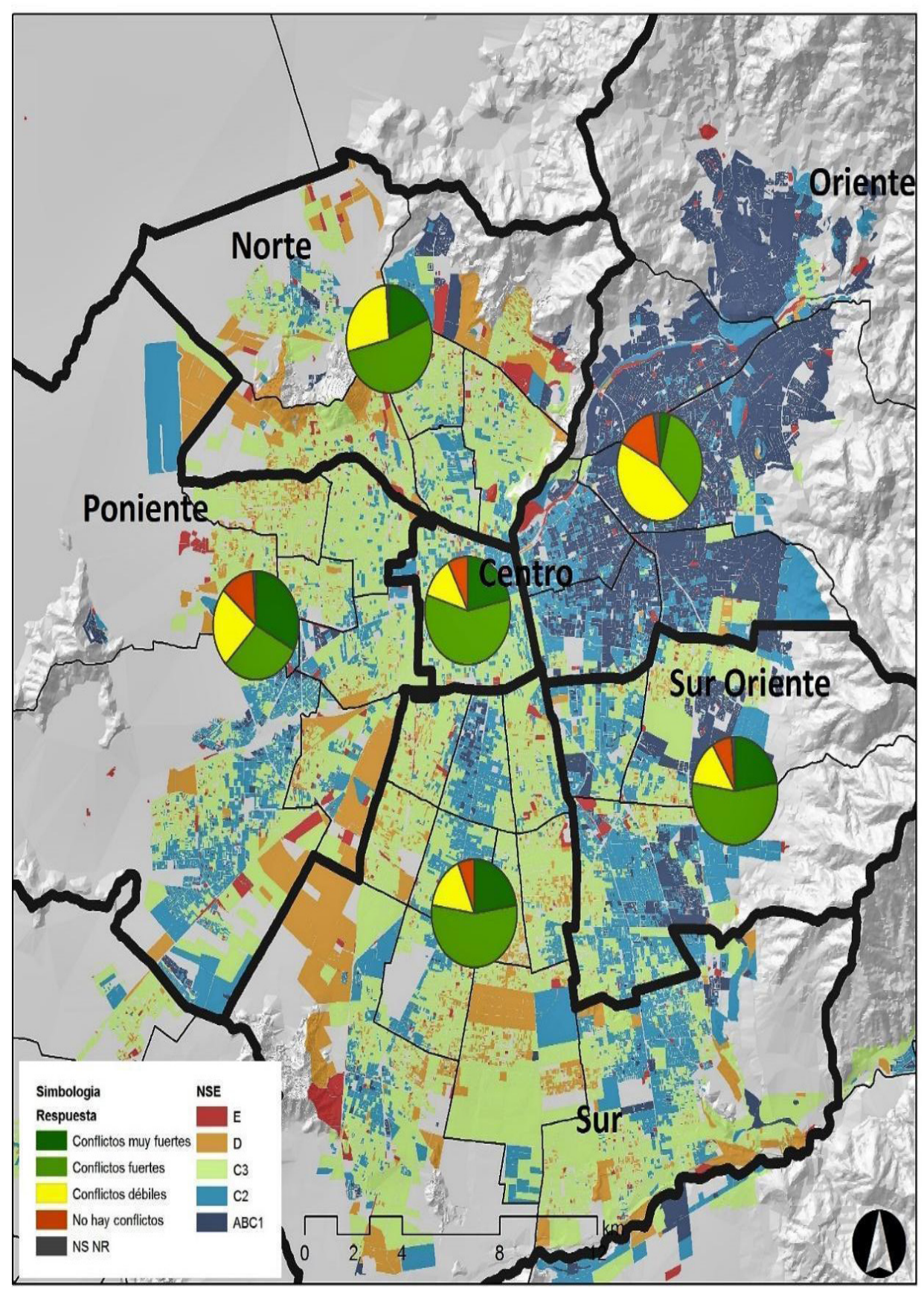

Fuente: El autor, 2017. 
Nótese que en la zona oriente, lugar donde habita la élite tomadora de decisiones, casi el 70\% de la población pensaba que hay conflictos débiles o simplemente no hay conflictos. El resultado de la medición para esta misma pregunta a nivel de Latinobarómetro, por su parte, arrojó que el $76 \%$ de la población del continente señala que existen conflictos fuertes o muy fuertes.

Por lo tanto, ya en el 2017 existía una alerta respecto a la percepción diferenciada de los conflictos en la sociedad chilena por territorio, lo que podría estar en la base del comentario del presidente Piñera respecto a que "definitivamente vivimos algo que nadie anticipó, que nunca había ocurrido" (Diario La Tercera, 2019) en relación al 18 de octubre de 2019. Las investigaciones apuntaban a un estallido social, y muchos nos preguntábamos por qué no había explotado antes del 2019. La respuesta está en que la mayoría de la élite habita en la zona oriente de Santiago, en un espacio clausurado y sin posibilidad de vínculo con el resto de la ciudad, los que, como queda en evidencia en este gráfico, percibían que existía menor conflicto entre ricos y pobres que el resto de la ciudad.

\section{Conflicto entre clase trabajadora y clase media}

Esta pregunta fue realizada también por el Instituto de Investigación en Ciencias Sociales de la UDP (Universidad Diego Portales) en el año 2014, a nivel nacional el resultado mostraba que el 54,5\% de la población encuestada a nivel nacional consideraba que existían conflictos fuertes o muy fuertes entre la clase trabajadora y la clase media en Chile, cuestión que debió alarmar a cualquiera que leyera el informe, no obstante, el dato pasó desapercibido y no hubo, ni hay una política pública para revertir la situación. Esta pregunta no hacer referencia a clases en las antípodas, como sería preguntar por conflicto entre clase empresarial y clase trabajadora y aún así queda en evidencia la percepción de distanciamiento entre clases.

Al aplicar la encuesta en las diferentes zonas de la ciudad de Santiago queda en evidencia que ese $54,5 \%$ promedio nacional, esconde una realidad bastante más compleja a señalar, la absoluta desconexión, al menos en la percepción de conflictos, de los habitantes de la zona oriente respecto al resto de la ciudad. Esto también puede explicar lo sucedido el 18 de octubre y el estupor de las élites económicas, políticas y administradoras de justicia, ante estos hechos, la mejor expresión de esto el filtrado audio de la esposa del presidente que señala ante lo ocurrido el 18 de octubre “Estamos absolutamente sobrepasados, es como una invasión extranjera, alienígena, no sé cómo se dice" (BBC, 2019). La élite encerrada en el sector oriente, no alcanza a dimensionar el conflicto.

Gráfico 2 - En todos los países hay diferencias o incluso conflictos entre diferentes grupos sociales.

En su opinión, ¿Cuánto conflicto hay en Chile entre la clase trabajadora y la clase media?

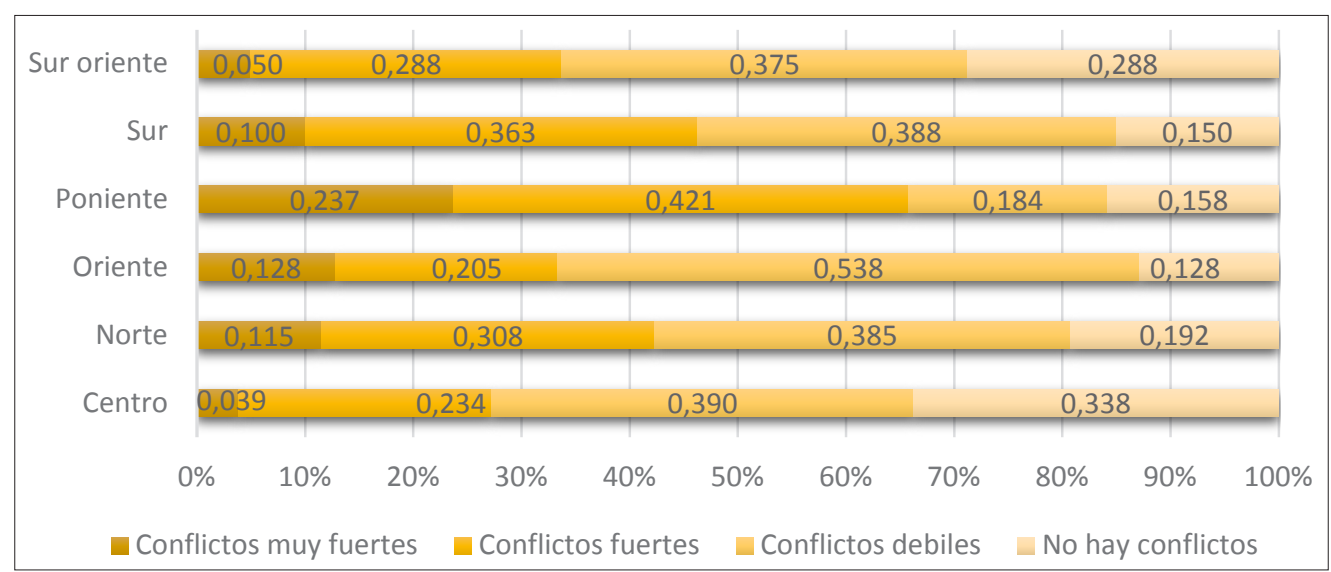

Fuente: El autor, 2016. 
Como queda en evidencia, y muy similar a los resultados de la UDP (2014) el 57,9\% de la población responde que en Chile no hay conflicto o hay conflictos débiles. Sin embargo, a desagregar por zona existe una notoria diferencia ya que el 54\% de la zona oriente asegura que en Chile los conflictos entre la clase trabajadora y clase media son débiles, versus, el 38\% de la población encuestada en la zona norte, el 39\% de la zona centro, el 39\% de la zona sur, y el 38\% de la zona suroriente.

\section{Conflictos por territorio la zona oriente y el resto de la ciudad}

Este el resultado que evidencia de modo más claro la diferencia de percepción de los conflictos entre los habitantes de la zona oriente y los habitantes del resto de la ciudad.

Gráfico 3 - En todos los países hay diferencias o incluso conflictos entre diferentes grupos sociales. En su opinión, ¿Cuánto conflicto hay en Chile entre la gente que vive en la zona oriente y la gente de otras comunas?

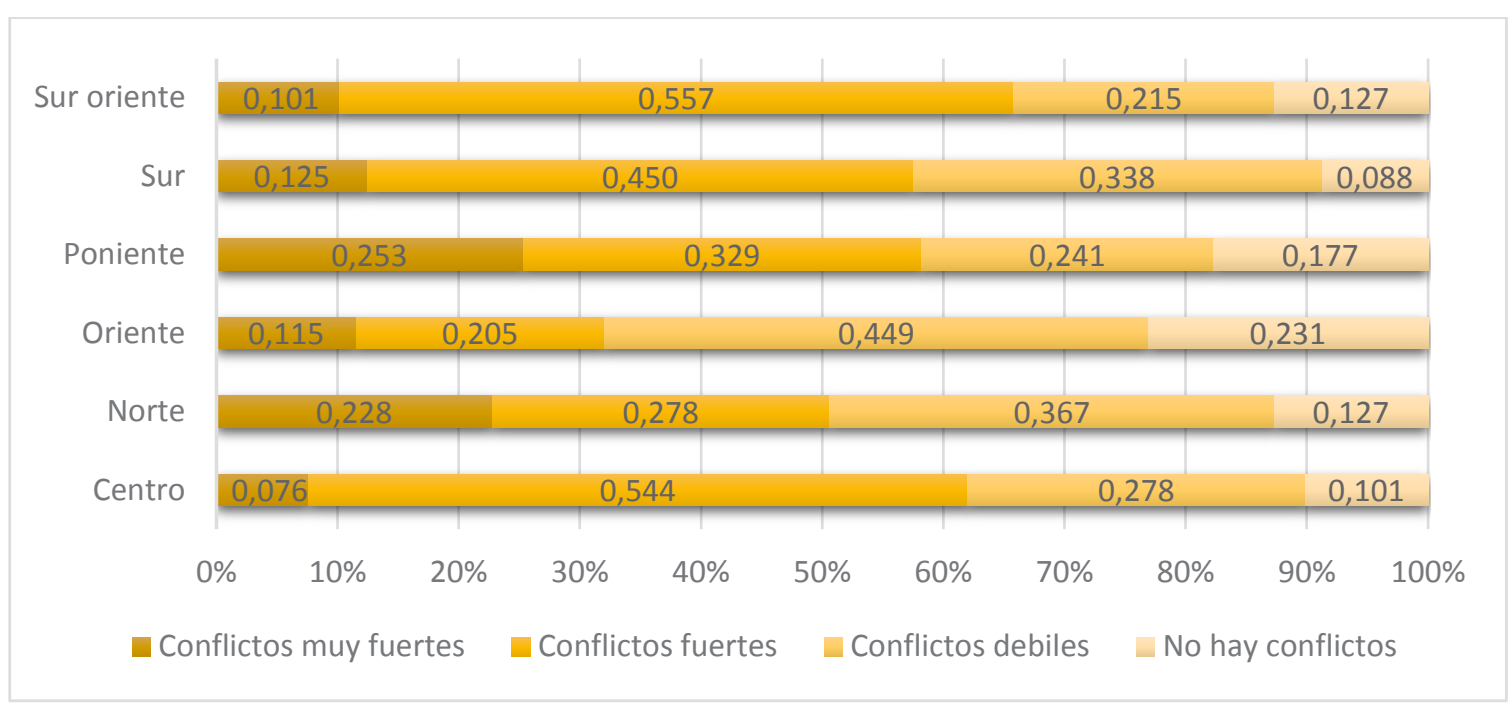

Fuente: El autor, 2016.

Enmarcando el conflicto en una dimensión territorial, el 84,9\% de la población encuestada, percibe que hay algún nivel de conflicto entre la gente que vive en la zona oriente y la gente que vive en el resto de las comunas. En particular, un 53,8\% de la población encuestada, percibe que hay conflictos fuertes o muy fuertes, un $31,1 \%$ percibe que hay conflictos, aunque débiles y solo un $14 \%$ señala que no hay conflictos.

A nivel zonal, la zona oriente difiere del resto, de modo similar al primer ítem, dado que sólo un $44 \%$, señala que hay conflictos, pero estos son débiles y el $22 \%$ señala que no hay conflictos, siendo esto un porcentaje superior al del resto de las zonas, las cuales perciben conflictos fuertes o muy fuertes en un $62 \%$ de la zona centro, 50\% de la zona norte, 58\% de la zona poniente, 58\% de la zona sur y $66 \%$ de la zona suroriente. 


\section{Conflicto entre los cuicos y los flaites}

En Chile, como en la mayoría de los países del mundo, existe una forma despectiva de tratarse entre clases sociales. Por una parte están los "flaites", concepto generalmente utilizado por la clase alta para referirse a una "persona de clase social baja y de comportamiento extravagante, y que es relacionada generalmente con el mundo delictual" (Rojas, 2015), que se viste de modo particularmente ostentoso y es fácilmente identificable en la sociedad chilena. Se suele utilizar el concepto de "flaite" también como un adjetivo que se utiliza como "sinónimo de "rasca" (otro concepto muy chileno), "ordinario", "maleducado" o "incivilizado". En este caso, el sujeto y su comportamiento particular se convierten en un adjetivo, en un vocablo para manifestar algo desagradable y/o cuestionable dentro de una sociedad normada" (González-Traslaviña, 2014). Este concepto viene a reemplazar el de "roto" de amplio uso para referirse a personas marginales y marginadas de la época colonial.

Por otra parte, se encuentran los "cuicos" concepto utilizado peyorativamente por las clases más pobres para referirse a las personas de clase alta, en las clases bajas los "cuicos" son personas pertenecientes a la clase alta, adinerados y que poseen cierto capital cultural específico que les permite acceder a espacios privilegiados y de poder. Este concepto en la clase alta es reemplazado por "pituco" y hace referencia a alguien cursi, presumido o presuntuoso más que a alguien que accede al poder, obviamente la mirada recursiva de la elite no permite identificar este aspecto dado que todos tienen de una u otra manera acceso al poder.

En esta investigación quisimos saber cómo percibían las personas encuestadas en los diversos territorios el conflicto entre estos dos grupos.

En el plano que se presenta a continuación se puede ver que el 79\% de los encuestados piensa que en Chile hay conflictos muy fuertes o fuertes entre la gente "cuica" y la gente "flaite". El 94\% de las personas que respondieron la encuesta en la zona norte, el 90\% de las de la zona suroriente, el $87 \%$ de la zona sur, el $84 \%$ de la zona centro, el $65 \%$ de la zona poniente y el $59 \%$ de la zona oriente, declara que en Chile hay conflictos muy fuertes o fuertes. En una línea similar al resto de los ítems, la zona oriente presenta una mayor percepción de estos conflictos como débil o inexistente mientras que el resto de las zonas, los conflictos son percibidos como fuertes o muy fuertes en mayor medida. 
Ilustración 4 - Conflicto entre la gente "cuica" y los "flaites.

\section{En Todos los países hay diferencias o incluso conflictos entre diferentes grupos sociales. En su opinión ¿Cuanto conflicto hay entre: la gente "cuica" y los "flaites"}

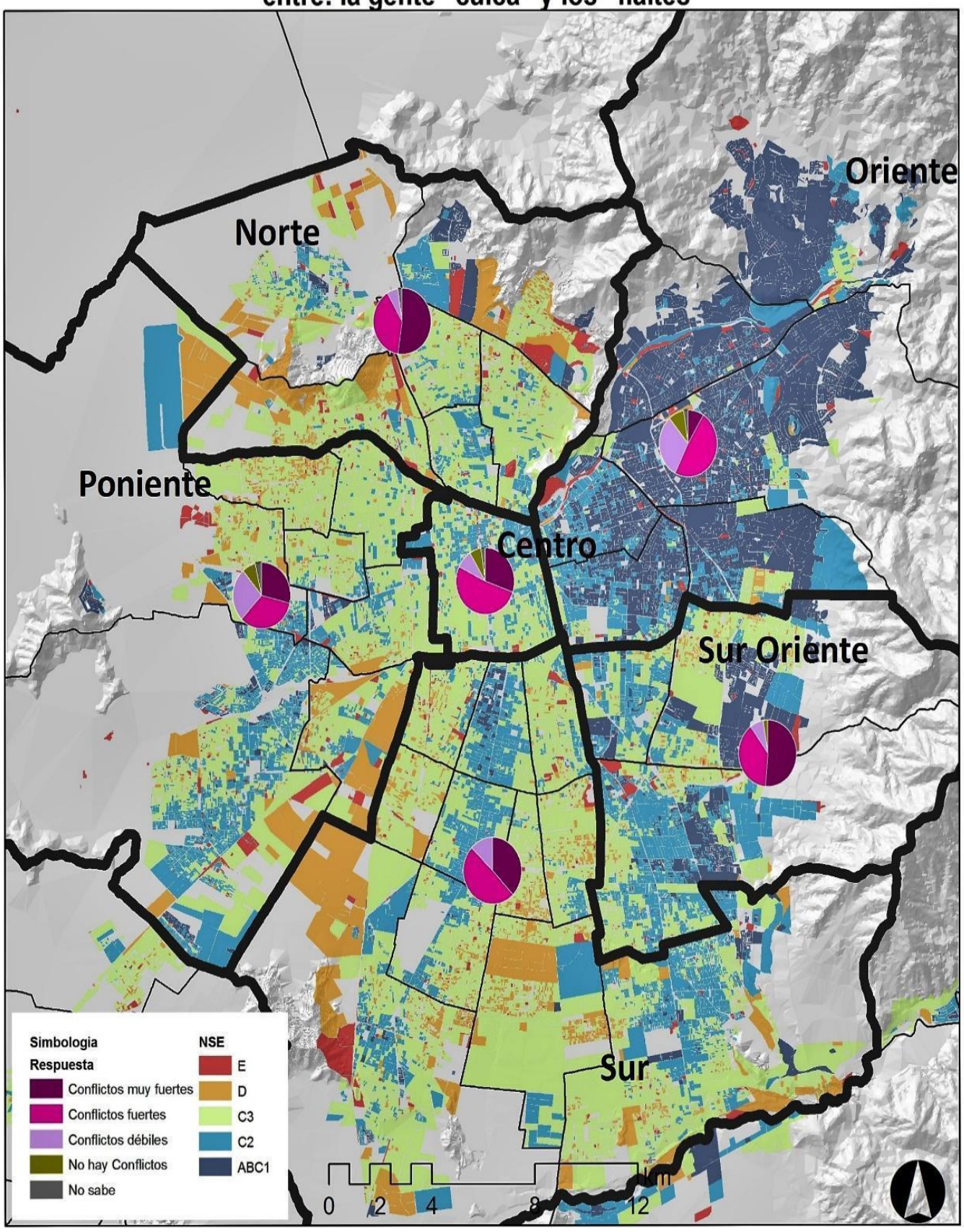

Fuente: El autor, 2016.

\section{Indicador Compuesto, Percepción de Conflicto}

A través de la producción de un Indicador Compuesto por Rotación Percepción de Conflicto con los ítems anteriormente expuesto se pueden observar diferencias sistemáticas entre la zona oriente y el resto de la urbe. Esto se ve en la representación gráfica de la distribución de esta 
variable compuesta. Por lo tanto, se aprueba la hipótesis que señala que hay relación entre la zona de residencia y percepción de conflicto y que en la zona oriente hay una menor percepción de conflicto que en el resto de las zonas estudiadas.

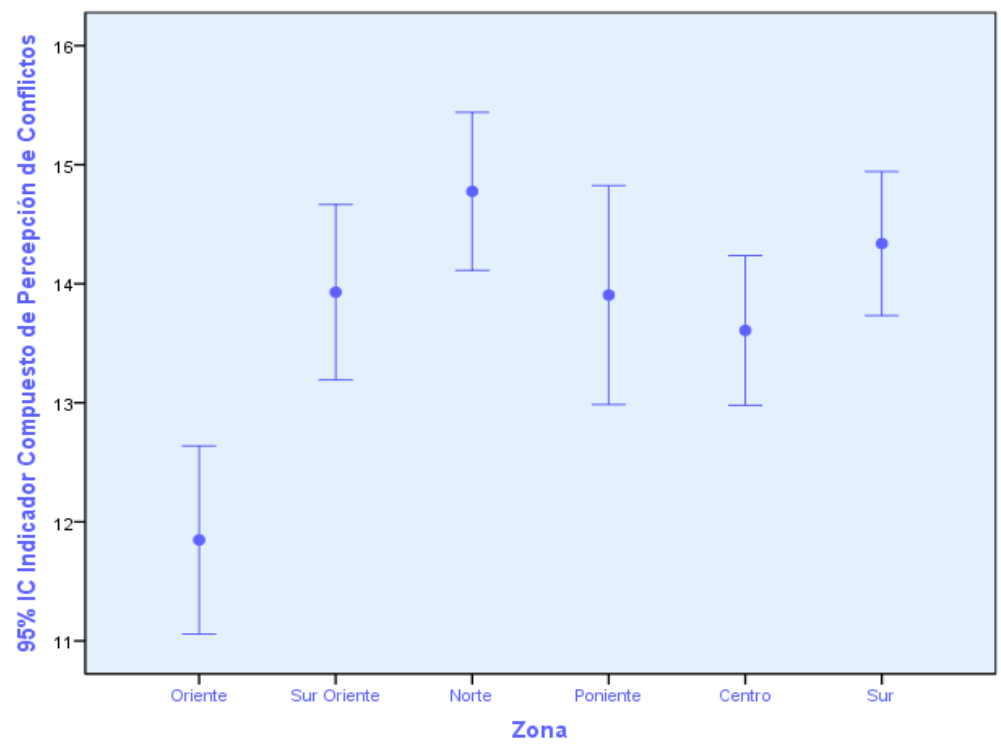

Fuente: El autor, 2018.

En el caso del índice compuesto de percepción de privilegios (que va de 4 a 16), se puede ver que la media de la zona oriente, 11.85, es menor a la del resto de las zonas, es decir, en promedio, los habitantes de zona oriente encuestados tienen la percepción de que el conflicto hoy en Chile es menor respecto a la percepción que tienen los habitantes el resto de las zonas.

Esto refuerza varias hipótesis, la primera es que efectivamente los habitantes de la zona oriente, en general con mayor capital social, económico y cultural, los sucesos ocurridos el 18 de octubre los pilló desprevenidos, por lo tanto, no fueron capaces de comprender y menos de actuar ante la contingencia. Los motivos de este desconocimiento están en el plano personal, no hacer un intento por conocer el resto de la ciudad y del país, pero por sobre todo están en el plano socioterritorial, en Chile la configuración de los territorios representa claramente la fragmentación social existente en el país.

\begin{tabular}{|c|c|c|c|c|c|c|c|c|}
\hline \multicolumn{9}{|c|}{ Descriptivos } \\
\hline \multicolumn{9}{|c|}{ Indicador Compuesto de Percepción de Conflicto } \\
\hline & \multirow[t]{2}{*}{$\mathrm{N}$} & \multirow[t]{2}{*}{ Media } & \multirow{2}{*}{$\begin{array}{l}\text { Desviación } \\
\text { típica }\end{array}$} & \multirow{2}{*}{$\begin{array}{l}\text { Error } \\
\text { típico }\end{array}$} & \multicolumn{2}{|c|}{$\begin{array}{l}\text { Intervalo de confianza para la } \\
\text { media al } 95 \%\end{array}$} & \multirow[t]{2}{*}{ Mínimo } & \multirow[t]{2}{*}{ Máximo } \\
\hline & & & & & Límite inferior & Límite superior & & \\
\hline Oriente & 79 & 11,85 & 3,527 & ,397 & 11,06 & 12,64 & 5 & 20 \\
\hline \begin{tabular}{|l|} 
Sur \\
Oriente \\
\end{tabular} & 71 & 13,93 & 3,114 & 370 & 13,19 & 14,67 & 5 & 20 \\
\hline Norte & 76 & 14,78 & 2,906 & ,333 & 14,11 & 15,44 & 8 & 20 \\
\hline Poniente & 74 & 13,91 & 3,970 & 461 & 12,99 & 14,83 & 5 & 20 \\
\hline Centro & 74 & 13,61 & 2,714 & ,315 & 12,98 & 14,24 & 5 & 19 \\
\hline Sur & 77 & 14,34 & 2,664 & 304 & 13,73 & 14,94 & 7 & 20 \\
\hline Total & 451 & 13,72 & 3,302 & ,155 & 13,42 & 14,03 & 5 & 20 \\
\hline
\end{tabular}


La hipótesis es también reforzada positivamente a través de un análisis ANOVA, conteniendo los diferentes componentes o fuentes de variabilidad, inter-grupos e intra-grupos, se obtiene un nivel de significancia inter-grupos menor a 0.05 , por lo tanto, se puede sostener que existe diferencia significativa entre zonas respecto a la percepción de conflicto.

ANOVA

Indicador Compuesto de Percepción de Conflicto

\begin{tabular}{|l|l|l|l|l|l|}
\hline & $\begin{array}{l}\text { Suma de } \\
\text { cuadrados }\end{array}$ & gl & $\begin{array}{l}\text { Media } \\
\text { cuadrática }\end{array}$ & F & Sig. \\
\hline Inter-grupos & 397,582 & 5 & 79,516 & 7,847 &, 000 \\
\hline Intra-grupos & 4509,216 & 445 & 10,133 & & \\
\hline Total & 4906,798 & 450 & & & \\
\hline
\end{tabular}

Dado que se encontraron diferencias significativas en ANOVA, se aplicó la prueba post hoc, HSD de Tukey para corroborar en qué zonas se encuentran estas diferencias.

Indicador Compuesto de Percepción de Conflicto

HSD de Tukeya,b

\begin{tabular}{|l|l|l|l|}
\hline \multirow{2}{*}{ Zona } & \multirow{2}{*}{$\mathrm{N}$} & \multicolumn{3}{l|}{ Subconjunto para alfa $=0.05$} \\
\cline { 3 - 4 } & & 1 & 2 \\
\hline \multirow{2}{*}{$\begin{array}{l}\text { Oriente } \\
\text { Centro }\end{array}$} & 79 & 11,85 & \\
\hline $\begin{array}{l}\text { Poniente } \\
\text { Sur Oriente }\end{array}$ & 74 & & 13,61 \\
\hline $\begin{array}{l}\text { Sur } \\
\text { Norte }\end{array}$ & 74 & & 13,91 \\
\hline Sig. & 71 & & 13,93 \\
\hline & 77 & 1,000 & 14,34 \\
\hline & 76 & & 14,78 \\
\hline
\end{tabular}

Se muestran las medias para los grupos en los subconjuntos homogéneos.

a. Usa el tamaño muestral de la media armónica $=75,080$.

b. Los tamaños de los grupos no son iguales. Se utilizará la media armónica de los tamaños de los grupos. Los niveles de error de tipo I no están garantizados.

En este caso, se forman dos subconjuntos claramente diferenciados, uno formado por la zona oriente y el otro compuesto por el resto de las zonas de la ciudad, en el cual el primer subconjunto muestra una menor percepción de conflicto. Esto refuerza la idea que la zona oriente conforma un espacio de percepción diferenciada del resto de la ciudad.

Si al análisis se agrega el hecho que es en la zona oriente en donde habitan aquellos que concentran el poder económico, político, del conocimiento, poder judicial, poder ejecutivo y legislativo, se puede deducir por qué el 18 de octubre se produjo una movilización de una envergadura tal que hizo tambalear aún más la ya precaria la institucionalidad del país.

\section{Discusión}

De este modo, la distinción entre el sector oriente y el resto del Gran Santiago en los resultados de la escala de percepción de conflicto se suman al conjunto de distinciones territoriales materiales, políticas y económicas anteriormente mencionadas entre los sectores de la ciudad. 
En este sentido, la percepción de conflicto puede leerse como parte de los marcos interpretativos que implican estas distinciones y podrían ser leídos desde el aparato teórico de Pierre Bourdieu y Foucault. En esta línea, dentro del marco de la investigación doctoral en la que se encuentra este texto, propone los términos capital y habitus territorial para describir tanto los elementos objetivos e incorporados que se asocian con habitar y poseer un territorio, sea el acceso a servicios públicos, como oportunidades y contactos y el capital simbólico asociado al territorio mismo, denominado habitus territorial, íntimamente asociado a un habitus de clase, que asegura la reproducción de las mismas. Este habitus territorial vendría acompañado de una doxa, un marco de creencias comunes que funciona como marco de legitimación del conjunto de prácticas y relaciones con los demás (Bourdieu \& Eagleton, 2003).

El habitus territorial en el Gran Santiago sería sostenido por una doxa de la segregación que sostiene como creencia irreflexiva y naturalizada la existencia de "unos" y "otros" dependiendo del territorio habitado. La doxa del habitar segregado configura una opinión masificada que señala que el habitar segregado es natural y hasta "deseable", que se ha naturalizado el vivir con los iguales y obviar a los diferentes; esta doxa permite que se construya un entramado urbano que incluye espacios clausurados, condominios cerrados, carreteras que niegan al otro como la Costanera Norte que conecta la zona más adinerada de la ciudad con el aeropuerto y con éste, conecta esta zona con resto del mundo y, a pesar de que esa vía se encuentra emplazada en territorios muy pobres, las personas que van en los autos a alta velocidad no logran verlos a causa de sendas murallas, pensadas como "aislantes de ruido" pero que en la práctica operan como aislantes del "otro" dado que también aíslan la posibilidad de ver el entorno en el que habita la otredad.

Los territorios en sí mismos contienen además elementos que permiten que el habitar en unos y otros implique diversidad de jerarquías, esto basado en hechos objetivos y subjetividades. En cuanto a los hechos objetivos, se pueden mencionar diversas mediciones, similares al ya señalado IDH comunal, el que permite identificar a algunas comunas como comparables con Europa (Vitacura, Lo Barnechea, Las Condes, etc.) y otras comparables a países africanos (La Pintana, Puente Alto, Quilicura, etc.). En cuanto a las subjetividades, vivir en una comuna u otra establece una suerte de estatus social percibido y recubre a quienes habitan el territorio de un halo de estigma si se habita en comunas pobres, o de superioridad si se habitan en comunas ricas.

Esta construcción de una alteridad territorial, que desde los ítems trabajados aparece compuesta tanto por una identificación territorial como de clase, también se ha presentado en otras investigaciones similares. Por ejemplo, COES (2019) en el marco de la encuesta ELCOS 2016-2018. Como se señaló, los habitus territoriales diferenciados, sumado a las jerarquías de los territorios habitados, potencian la aparición de estigmas territoriales.

La percepción de estigmas territoriales se relacionaría tanto con el lugar habitado como con el nivel socioeconómico y educacional, en tanto los mayores niveles de percepción de valoración negativa aparecen en los quintiles más bajos, así como con menores niveles educativos, y en el caso de Santiago y otras urbes metropolitanas, estos se presentaría con una mayor intensidad y heterogeneidad que en otras zonas.

Es así cómo, tomando el concepto de doxa territorial, este no solo incluiría las creencias mismas respecto a quién pertenece a cada territorio, sino también creencias respecto a cómo uno es representado por aquellos que habitan otros lugares, los cuales, en el caso de las comunas con menor concentración de riquezas, aparecen como una percepción negativa. En este sentido, volviendo al concepto de doxa territorial, fuera de la zona oriente la construcción del "otro" implicaría no solo una posición jerárquica y su valoración asociada, sino también la construcción 
de un "otro" que me valora de forma negativa, lo cual podría servir para explicar las diferencias naturalizadas en cada territorio, respecto a percepción de conflicto.

La identificación de una clase con un territorio podría ser un elemento explicativo de la percepción diferenciada de conflicto, en tanto que Pérez (2013) argumenta que la consciencia de clase y la percepción de conflicto que necesariamente viene asociada a este según el autor, sigue siendo un prisma relevante para la lectura de lo político en Chile. Pérez describe cómo la percepción de conflicto de clases todavía se relaciona fuertemente con la posición estructural y tipo de trabajo en tanto que trabajadores agrícolas, trabajadores manuales calificados y no calificados mostrarían una mayor probabilidad de percibir niveles de conflicto fuertes, mientras que las clases de servicio altas y bajas presentan lo contrario. Esta caracterización respecto a la percepción del conflicto coincide con los resultados arrojados por esta investigación.

Sin embargo, añadiendo al concepto de doxa territorial, también podría ser útil considerar la noción de una illusio territorial y los efectos que esto podría tener en la constitución de los campos en la urbe. La illusio puede entenderse como la noción del capital y posiciones que se ponen en juego en un campo tienen valor, así como que las normas para disputarlo tienen sentido (Rowlands \& Rawolle, 2013). Este es un elemento crucial para la teoría de campos de Bourdieu, en tanto la capacidad de competir, transformar y usar el capital de maneras propias al mismo campo aparecen como una característica fundamental para establecer la delimitación de campos y subcampos específicos (Bourdieu, 1993). En particular, en una sociedad como la chilena en la cual las reformas neoliberales solo han recibido grandes desafíos en la última década (Undurraga, 2015), los modos para disputar el capital también estarían impregnados de estas lógicas. Para Foucault (2007) uno de los elementos centrales en las lógicas de gobierno de la segunda mitad del siglo $\mathrm{XX}$, y en particular los estados neoliberales, es el modo en que los sujetos dejan de ser un hecho pasivo del mercado, y deben ser gobernados como sujetos de empresa que garanticen el máximo nivel de competencia, y que, a través de conceptos como el capital humano, cada individuo sea capaz de volverse un espacio de inversión en sí mismo. Siguiendo esta línea, Rose (1999) elabora sobre cómo este cambio en la lógica de gobierno viene acompañado de un mandato ethopolitico, en el cual los sujetos deben volverse responsables de su propia rentabilidad y empleabilidad a través de distintas políticas de reforma personal. En este sentido, y retomando las ideas de Beck (1986), Castell (1990) y Bauman (2003), los sujetos deben hacerse responsables del riesgo, de la liquidez, de la inestabilidad y precarización de sus vidas, en suma, deben asumir los riesgos que la inversión en sí mismos conlleva. Y como lo han señalado estos riesgos son distribuidos inequitativamente en el territorio, favoreciendo a la zona oriente de Santiago.

Teniendo lo anterior en mente, las diferencias respecto a la percepción del conflicto podrían indicar que las "reglas del juego", que no afectan a todos por igual, no aparecen como transversalmente legitimadas en tanto el conflicto aparece precisamente como una forma explícita de disputa por el capital dentro de un campo. La diferencia en la percepción del conflicto y su intensidad en un campo podría asociarse a la estabilidad percibida de las normas del juego dentro de éste.

Es así cómo, mientras que en la zona oriente la percepción de conflicto es menor, dado que, considerando las diferencias de clase entre comunas, tanto el capital como aquellos con los que se disputa son parte de un mismo campo territorial, con una doxa y habitus asociados, el resto de la urbe, que en el esquema tradicional de Bourdieu aparecen como sujetos dominados con el habitus correspondientes, competirán por tener acceso a este subcampo del que son vedados a pesar de también poseer una doxa que legitima su exclusión. En este sentido, Arango (2002) 
elaborando sobre la participación de grupos dominados en subcampos específicos menciona cómo la relación con la illusio para éstos aparece atrapada en una disyuntiva, en tanto, la mayoría de los campos especializados son de participación casi exclusiva de la clase dominante, y por lo tanto la dominación simbólica en parte se constituye por la exclusión de los dominados del área de juego. Aquellos que de alguna manera logran participar se ven con la disyuntiva de no poder irrumpir con la illusio del campo, que en parte se sostiene a través de su propia exclusión, más allá de ciertos límites en tanto amenazarían la constitución misma del campo. En esta línea, la noción de conflicto podría cumplir el rol de desnaturalizar las distinciones de clase y distribución del capital, y posibilitar la disputa de éste, sin anular las condiciones de existencia misma del campo.

Esta disputa podría leerse desde lo que Goméz (2008) denomina como un conflicto cultural, el cual específicamente se referiría a aquellos conflictos donde la matriz cultural es utilizada para visibilizar la arbitrariedad de las reglas del juego político a través de un esquema simbólico que representaría los varios elementos en disputa que posteriormente servirían para la proposición de nuevas reglas para el juego político. Goméz (2008) resalta cómo esta forma de conflicto difiere de los conflictos políticos tradicionales, en tanto este se basaría en la generación de consensos a través de las reglas ya existentes y que tendría el efecto de volver los elementos de conflicto como elementos latentes, en cambio, el conflicto cultural sería inteligible a las formas políticas tradicionales y buscaría instaurar reglas y elementos culturales distintos. De este modo, de manera similar a la dicotomía entre deliberación y conflicto, la percepción de conflicto, así como sus significados culturales, serían distintos entre aquellos que estén en una posición en la cual puedan disputar la reglas del juego político y hacer explícitos los elementos latentes de éste con aquellos que debido a su posición estructural se verían beneficiados por estas normas, y para mantener aquella posición deberían minimizar el conflicto para volverlo legible dentro de las reglas existentes del campo para posibilitar su absorción.

De este modo, podríamos observar un mayor nivel de conflicto percibido en aquellos espacios donde las instituciones políticas dejan de aparecer como actores legítimos de diálogo, y aquellos conflictos que estas marcarían como latentes se vuelven explícitos como sucedió el 18 de octubre. Y en el inverso, en aquellos espacios donde las instituciones políticas poseen mayor legitimidad, y en los cuales se posee mayor acceso a ellas, debiese existir una menor percepción de conflicto, esto explicaría el estupor y desconcierto de los habitantes de la zona oriente y por tanto de los poderosos ante el estallido social.

Efectivamente, dentro del marco más amplio de esta investigación, en el sector oriente se concentra gran parte del poder político y económico en la urbe, así como una mayor percepción de legitimidad de las instituciones, aunque es importante notar que, en términos de confianza en estas, la urbe es relativamente homogénea mientras que el nivel de satisfacción con las mismas es más bajo en la zona oriente. Esto podría construirse como una contradicción aparente tanto con los datos anteriormente mencionados como con esta interpretación respecto a la percepción de conflicto.

Sin embargo, esto podría deberse a un imaginario distinto respecto a las capacidades y rol de las mismas. Por ejemplo, utilizando la comparación de González (2014) respecto al rol del conflicto en Habermas y Mouffe, podemos enmarcar la percepción del conflicto, así como su rol en la sociedad dentro de una dicotomía, en la cual para el modelo Habermasiano deliberativo, estos aparecen como una eventualidad, posiblemente insalvable, dentro de los procesos de diálogo democráticos para establecer consensos, pero no serían una característica fundamental de lo político. Por otro lado, desde Mouffe, bebiendo de Schmidt, el conflicto aparece como 
parte de un antagonismo fundamental en tanto toda comunidad política necesita marcar una distinción con otro y un límite de aquello que es aceptable dentro de esta y que para Mouffe, debe ser transformado en un agonismo a través de las prácticas e instituciones democráticas, entendiendo agonismo como aquellos aspectos que pudiere tener el conflicto, el que en una democracia debiera ser aceptado, debido a su innegable existencia, y encauzado positivamente. En este sentido, las normas del juego, el rol del conflicto y su intensidad entre estos marcos son fundamentalmente diferentes, en tanto en el primero, el conflicto aparecería como un preludio al diálogo y su posible resolución, mientras que en el segundo sería un problema continuo que solo podría ser mitigado. En un espacio en el cual las instituciones carecen de legitimidad, y por lo tanto no pueden constituirse como actores legítimos para el diálogo, cabe pensar que podrían moverse a una concepción más cercana a Mouffe respecto al conflicto, enmarcándolo como un elemento explícito y sin resolución, no quedando nada más entonces que encausarlo positivamente por medio de un diálogo entre todos los actores involucrados, el no hacerlo pudiere implicar la agudización del mismo. A modo de ejemplo, lo que sucedido en Chile el 18 de octubre de 2019, momento más álgido de un conflicto previamente existente pero no abordado por una institucionalidad, la que negó por años el diálogo entre actores y fue capturada por un grupo que, a pesar de pertenecer a diversos y antagónicos partidos políticos, habitaba en la zona oriente de Santiago, sin permitir el diálogo con los "otros" habitantes de otros territorios.

\section{Conclusión}

En resumen, las primeras décadas del siglo XXI ha traído consigo cambios importantes en la manera en quese constituye la sociedad y ésta se piensa a sí misma; en consecuencia, ha habido cambios en las maneras que se entiende y dialoga el conflicto. Estas transformaciones tienen un componente territorial crucial ser comprendidas, en tanto éste se enmarca como un espacio constituido y constituyente de las relaciones de poder que ocurren en el mismo. En particular, el caso de Santiago de Chile aparece como un espacio que ha vivido una serie de radicales transformaciones económicas, políticas y sociales que han tenido como efecto niveles considerables de segregación, así como diferencias en calidad de vida. Como parte del marco de una investigación más amplia sobre la concentración socio-espacial del poder y la legitimidad del orden social se realizó una encuesta en las comunas del Gran Santiago. En este texto se trabajó sobre la dimensión de percepción de conflicto de la encuesta, la cual se centró en la percepción de conflictos de clase y de territorio. Un análisis de estos datos muestra diferencias significativas entre la zona oriente y el resto de las zonas de la urbe, en tanto que, en ésta tanto la percepción del conflicto como la intensidad del mismo son menores.

Estas diferencias podrían estar asociadas a un habitus y doxa territoriales que enmarcan las creencias alrededor de quienes viven en el territorio, los bienes y servicios a los que tienen acceso y la manera en que éstos se disputan. En esta línea, estas diferencias se suman a otras que han sido reportadas en investigaciones similares, tales como la percepción de estigmatización percibida de gente ajena a la zona oriente. Además, se propone que la percepción de conflicto se asocia no solo a estas métricas, sino que también podría relacionarse con la manera en la que entiende el conflicto mismo y el rol de las instituciones democráticas frente a éste, tanto como mecanismos de resolución como de invisibilización.

Se plantea que el 18 de octubre de 2019, en parte en consecuencia de esta excesiva fragmentación socioeconómica, pero también sobre todo territorial, en tanto el distanciamiento socioeconómico se ve agudizado por el distanciamiento territorial y la concentración y clausura de la 
clase dominante en la zona oriente de la ciudad. Esto abre varias posibilidades para investigaciones posteriores respecto a qué efectos tienen estas diferencias y cómo se articulan en discursos y prácticas particulares dentro del territorio. En particular, investigaciones cualitativas enfocadas en las distintas nociones de conflicto, así como sus posibles vías de resolución, podrían entregar importantes elementos interpretativos para entender cómo se subjetiva la exclusión y el conflicto en Chile. De la misma manera, el modo en que la percepción de conflicto se asocia a dimensiones como la estigmatización percibida de la comuna, o la calidad de vida en la misma también podrían ser áreas relevantes de estudio. En este sentido, los conceptos de habitus e illusio territorial podrían servir como elementos articuladores para esta y otras líneas de investigación.

\section{Glosario}

-NSE: Las siglas significan "Niveles Socioeconómicos”, y fue creado por la Asociación Mexicana de agencias de Inteligencia de Mercado y Opinión (AMAI) como una regla basada en un modelo estadístico que clasifica y agrupa los hogares en siete niveles, de acuerdo a su capacidad de satisfacer las necesidades de sus integrantes, lo que determina calidad de vida y bienestar.

-SPSS: Las siglas significan “Producto de Estadística y Solución de Servicio”, el cual es un Software de la IBM diseñado para las Ciencias Sociales, por lo que utiliza una amplia gama de análisis estadísticos, siendo capaz de analizar datos para la generación de tablas y gráficos con data de alta complejidad.

\section{Referencias}

ARANGO, L. Sobre dominación y luchas: clase y género en el programa de Bourdieu. Revista colombiana de sociología, 7(1), pp. 99-118. 2002

BANCO CENTRAL CHILE. Encuesta Financiera de Hogares: Metodología y Principales

Resultados EFH 2011-12.2012. Recuperado de http://www.bcentral.cl/estadisticas-economicas/ financierahogares/pdf/Resultados_EFH_2011-12.pdf.

BAUMAN, Z. La Modernidad Líquida. México: Fondo de cultura económica. 2003.

BECK, U. La sociedad en riesgo, hacia una nueva modernidad. Barcelona: PAIDÓS. 1986.

BOURDIEU, P. The Field of Cultural Production: Essays on Art and Literature. Cambridge: Polity Press. 1993.

BOURDIEU, P., y Eagleton, T. “Doxa y vida cotidiana”. En S. Zizek (Ed), Ideología: un mapa de la cuestión. pp. 295-308. Madrid: Fondo de Cultura Económica. 2003.

CASTAÑEDA, P. El endeudamiento como problemática social emergente. Concepción: Universidad Bio-Bio. 2012.

COES. Radiografía del cambio social Análisis de Resultados Longitudinales Estudio Longitudinal Social de Chile ELSOC 2016-2018. 2019. Recuperado de https://drive.google.com/file/d/0B3Y48LCzP OdQb2dXd2plaHpUcHJxcUlsYTlrblNpdDBheENj/view

CORREA, J. El Valor del suelo como reflejo y reproductor de las desigualdades Centro de Producción del Espacio. 2020 
DE CERTEAU, M. La invención de lo cotidiano: Artes de hacer I. Vol. 1. Pescador, A. (Trad). México D.F.: Universidad Iberoamericana. Originalmente publicado en 1990. 2002.

FACULTAD DE ECONOMÍA Y NEGOCIOS, UNIVERSIDAD SAN SEBASTIÁN \& EQUIFAX. XIII Informe de Deuda Personal Deudores Morosos. Santiago: Universidad San Sebastián. 2016.

FLEET, N. Movimiento estudiantil y transformaciones sociales en Chile: una perspectiva sociológica. Polis 30. 2012. Recuperado de http://journals.openedition.org/polis/2152

FOUCAULT, M. Of Other Spaces: Utopias and Heterotopias. (J. Miskowiec, Trad) Architecture/ Mouvement/Continuité, 5. 1984. Recuperado de http://web.mit.edu/allanmc/www/foucault1.pdf

FOUCAULT, M. Nacimiento de la Biopolítica: Curso en el College de France (1978-1979) (H. Pons, Trans.). Buenos Aires, Fondo de Cultura Económica. 2007.

GARRETÓN, M. La sociedad en que vivi(re)mos: introducción sociológica al cambio de siglo. Santiago: LOM Ediciones. 2000.

GÓMEZ. A. Sobre el carácter cultural de la emergencia de conflictos sociales en Chile. Revista Mad, 18, pp. 20-37. 2008. DOI: 10.5354/0718-0527.2011.13908

GONZÁLEZ, J., Habermas y Mouffe: La democracia entre consenso y conflicto. En J. Franzé (Ed)

Democracia: ¿consenso o conflicto? Agonismo y teoría deliberativa en la política contemporánea, Madrid: Catarata, pp. 63-90.2014.

HARVEY, D. Seventeen Contradictions and the End of Capitalism. Reprint Edition, 2014.

INE. ACTUALIZACIÓN DE POBLACIÓN 2002-2012 Y PROYECCIONES 2013-2020. 2016. Recuperado de http://www.ine.cl/canales/chile_estadistico/familias/demograficas_vitales.php

KORZENIEWICZ, R. P. Unveiling Inequality, a World Historical Perspective. Nueva York: Fundation, Russell Sage. 2009.

MASSEY, D. Politics and Space/Time. New Left Review, I (196), 65-84. 1992.

MIDEPLAN. Resultados encuesta CASEN 2009. 2010. Recuperado de https://www.bcn.cl/siit/ actualidad-territorial-19-5-2015/casen_2009

MIDEPLAN \& DICTUC-PUC. Encuesta origen destino 1991.1991. Recuperado de http://www.sectra. gob.cl/biblioteca/listado_corto1.asp

MINISTERIO DE TRANSPORTE Y TELECOMUNICACIONES, SECTRA - Observatorio Social, UAH. Informe ejecutivo, encuesta origen y destino de viajes 2012. Santiago de Chile: Ministerio de Transporte y Telecomunicaciones. 2012. Recuperado de http://www.sectra.gob.cl/biblioteca/detalle1.asp?mfn=3253

MINISTERIO DE TRANSPORTE Y TELECOMUNICAIONES, SECTRA-PUC. Encuesta origen y destino de viajes 2001. 2001. Santiago: SECTRA. Recuperado de http://www.subtrans.gob.cl/subtrans/doc/ estadisticasEOD2001_Informe_Difusion.pd

PÉREZ, P. Encontrando lo que nunca estuvo perdido. Conciencia de clase y conflicto de clases en el régimen neoliberal chileno. Revista de sociología. 28, pp. 83-111. 2013. DOI: 10.5354/0719529X.2013.30716

PNUD. Las Trayectorias del Desarrollo Humano en las Comunas de Chile (1994-2003) (Vol. $\mathrm{N}^{\circ}$ 11).2004. Recuperado de http://www.plataformacaldera.cl/biblioteca/589/articles-66229_ documento.pdf 
ROSE, N. Powers of Freedom: Reframing Political Thought [Poderes de Libertad: Reenmarcando el pensamiento político] Cambridge: Cambridge University Press.1999.

ROWLANDS, J., \& Rawolle, S. Neoliberalism is not a theory of everything: A Bourdieuian analysis of illusio in educational research. Critical Studies in Education, 54 (3), pp. 260-272. 2013. DOI: 10.1080/17508487.2013.830631

SBIF. Informe de endeudamiento 2016. Santiago: SBIF. 2016. Recuperado de https://www.sbif.cl/ sbifweb/servlet/Publicaciones?indice=15.3\&idPublicacion=309\&idContenido=11422\&idCategoria=2511

SEPÚLVEDA, M. El orden de mercado y la hegemonía neoliberal en Chile. La regulación de la vida social por las lógicas mercantiles. Historia 396,6(2), 399-429. 2016. Recuperado de http://www.historia396.cl/ index.php/historia396/article/view/96

SPRINTIGN, S. The handbook of neoliberalism. Ed. Routledge International Handbooks, 2016.

UNDURRAGA, T. Neoliberalism in Argentina and Chile: common antecedents, divergent paths. Revista de Sociología e Política, 23 (55), 11-34. 2015. DOI: 10.1590/1678-987315235502

VARGAS-AGUIRRE, M. Concentración territorial del poder, segregación socio - espacial y legitimidad del orden. El caso de Santiago de Chile. Tesis para optar al grado académico de Doctora en Ciencias Sociales, Universidad de Chile, 2018.

WACQUANT, L. Designing urban seclusion in the twenty-first century: The Class 2009 Roth-Symonds Lecture. Perspecta, 43, 164-175. 2010. Recuperado de www.jstor.org/stable/41680282

WALLERSTEIN, I. The Modern World-System IV: Centrist Liberalism Triumphant, 1789-1914, California, University of California Press, 2013.

WALLERSTEIN, I. Análisis del sistema mundo, una introducción. Madrid, Editorial Siglo XXI, 2005. 